\title{
Efficacy and Safety of Rivaroxaban versus Warfarin for the Treatment of Acute Pulmonary Embolism: A Real-World Study
}

\author{
Yan Huang, ${ }^{1,2}$ Linli Duan, ${ }^{1,3}$ Wenjun He, ${ }^{1}$ Cheng Hong, ${ }^{1}$ Yehui Guo, ${ }^{1}$ Xinni Wang, \\ Nuofu Zhang, ${ }^{1}$ Yanghang Chen, ${ }^{1}$ Tao Wang, ${ }^{1}$ Jian Wang $(1),{ }^{1}$ and Chunli Liu $\mathbb{1}^{1}$ \\ ${ }^{1}$ State Key Laboratory of Respiratory Disease, National Clinical Research Center for Respiratory Disease, Guangzhou Institute of \\ Respiratory Health, The First Affiliated Hospital of Guangzhou Medical University, Guangzhou, Guangdong 510120, China \\ ${ }^{2}$ Xishuangbanna Dai Autonomous Prefecture People's Hospital, Xishuangbanna Dai Autonomous, Yunnan, China \\ ${ }^{3}$ The Second Affiliated Hospital of Guangzhou Medical University, Guangzhou, Guangdong, China
}

Correspondence should be addressed to Jian Wang; jwang31@jhmi.edu and Chunli Liu; chunli.liu@aliyun.com

Yan Huang, Linli Duan, and Wenjun He contributed equally to this work.

Received 17 October 2019; Revised 25 May 2020; Accepted 3 June 2020; Published 26 June 2020

Academic Editor: Consuelo Amantini

Copyright (C) 2020 Yan Huang et al. This is an open access article distributed under the Creative Commons Attribution License, which permits unrestricted use, distribution, and reproduction in any medium, provided the original work is properly cited.

Background. Pulmonary embolism (PE) is a life-threatening disease. Target-specific anticoagulant rivaroxaban is a direct factor Xa inhibitor that can be safely used without laboratory monitoring. Objective. To investigate the efficacy and safety of rivaroxaban versus warfarin for the treatment of acute pulmonary thromboembolism in real-world clinical practice. Method. This was a semiretrospective, semiprospective, and real-world trial involving 128 patients with acute symptomatic pulmonary embolism with or without active tumor or frailty. We compared rivaroxaban to the standard therapy consisting of low-molecular-weight heparin combined with warfarin. The primary efficacy outcome was absorption of thrombus. The principal safety outcome was bleeding episode. Results. There was no significant difference in thrombus absorption between rivaroxaban and standard therapy after 3-month treatment $(P=0.798,95 \%$ confidence interval $(\mathrm{CI}) 0.686$ to 1.336$)$ or more than 6-month treatment $(P=0.534$, $95 \%$ confidence interval (CI) 0.795 to 1.556). There was no decline in efficacy (including computed tomographic pulmonary angiography and recurrence) when the rivaroxaban dose was reduced to $10 \mathrm{mg}$ once daily after 3 months of administration. The ratio of patients without bleeding was $48.84 \%$ for rivaroxaban and $19.05 \%$ for standard therapy $(P=0.001)$. There was no significant difference in rivaroxaban monotherapy subgroups (including frail patients, tumor patients, and thrombolysis or nonthrombolysis at intermediate-high-risk patients). Conclusion. In this real-world study, the efficacy and safety of rivaroxaban alone was not different to standard therapy for pulmonary emboli absorption. With an extension in treatment duration, the rivaroxaban regimen had a higher efficacy and safety than standard therapy and there was no decline in treatment efficacy when the rivaroxaban dose was reduced to $10 \mathrm{mg}$ once daily.

\section{Introduction}

Pulmonary embolism (PE) is a disease with high morbidity and mortality [1]. Because the usage of rivaroxaban is increasing in actual clinical practice, it is necessary to compare the rivaroxaban monotherapy regimen with standard therapy [2]. Even though several guidelines for the treatment of acute pulmonary embolism with rivaroxaban have been published, there are many PE patients with specific clinical characteristics including frailty (e.g., elderly, low body weight), oncology, or at intermediate-high-risk. Among them, elderly patients have a high incidence of bleeding [3], which increases when coupled with warfarin treatment; however, there are no guidelines for reducing the anticoagulant dose for that population. For low-weight patients, the efficacy of novel oral anticoagulants (NOACs) depends on its plasma concentrations, which are closely related to body mass/BMI. A previous RCT study in the field of atrial fibrillation reported that most NOACs increased the rate of bleeding in low-weight patients; therefore, the dose of apixaban and 
edoxaban should be adjusted according to the body weight [4]. However, there is no evidence which informs the adjustment of the dose of rivaroxaban based on the body weight. Tumors are the strongest independent risk factors of allcause mortality in patients with venous thromboembolism (VTE) $[5,6]$. Because of the recent development of NOACs, there is almost no evidence on whether rivaroxaban can be used to prevent VTE in cancer patients. For intermediatehigh-risk patients, systemic thrombolysis improved the potential of hemodynamic disturbances and improved treatment outcomes concurrently [7]. However, the PEITHO study reported the rate of hemorrhagic stroke was increased by $2 \%$ after thrombolysis in intermediate-high-risk patients [8]. Furthermore, for intermediate-high-risk patients, thrombolysis can increase the efficacy and risk of bleeding, but there have been no reports on whether rivaroxaban can achieve the same effect as thrombolysis without increasing the risk of bleeding.

We previously compared rivaroxaban monotherapy with standard therapy based on gene testing to adjust the dose of warfarin [2]. The results showed that the efficacy of rivaroxaban monotherapy was better than standard therapy and had a lower incidence of bleeding. Because of the small sample size in our previous study, the current study continued the comparison of the two regimens in real-world clinical practice.

Our trial consisted of two parts: (1) comparison of the efficacy and safety of rivaroxaban and warfarin and (2) subgroup analysis of rivaroxaban, which contained (a) efficacy and safety of frail and nonfrail patients in the rivaroxaban regimen; (b) efficacy and safety of tumor and nontumor patients with pulmonary embolism; and (c) efficacy and safety of the rivaroxaban regimen and thrombolytic therapeutic regimen in intermediate-high-risk patients.

\section{Materials and Methods}

2.1. Patients. This study was approved by the Ethics Committee of The First Affiliated Hospital of Guangzhou Medical University (trial registration: this study has been registered at ClinicalTrials.gov: ChiCTR-TRC-14005223; 12 September 2014). Inclusion criteria were $\geq 18$ years, acute pulmonary embolism diagnosed by computed tomographic pulmonary angiography (CTPA), and ventilation/perfusion (V/Q), with treatment of rivaroxaban monotherapy or standard-therapy. Exclusion criteria were no thrombus in chronic pulmonary embolism patients, or patients who received thrombectomy or vena cava filter.

2.2. Assignment and Treatment Regimens. Patients assigned to the rivaroxaban group received $15 \mathrm{mg}$ twice daily for the first 3 weeks, $20 \mathrm{mg}$ once daily from 3 weeks to 3 months, and followed by $10 \mathrm{mg}$ once daily for long-term anticoagulation. Patients assigned to the standard-therapy group received a dose of warfarin of 3-5 $\mathrm{mg}$ once daily followed by low-molecular-weight heparin (LMWH) $0.1 \mathrm{ml}$ per $10 \mathrm{~kg}$ body weight twice daily. LMWH was discontinued when the international normalized ratio (INR) was 2.0-3.0 over consecutive days, and then, INR was detected once a week.
The dose of warfarin was adjusted to maintain an INR of 2.0-3.0, and the INR was detected once a month.

For rivaroxaban subgroups, frail patients included patients aged $>70$ years or weight $<45 \mathrm{~kg}$ or $\mathrm{BMI}<18$. Tumor patients were defined as patients with active tumors, including patients who were undergoing radiotherapy and chemotherapy or were postoperative. Patients in the third subgroup had thrombolysis or no thrombolysis and were at intermediate-high risk.

2.3. Assessments. The primary efficacy outcome was absorption of thrombus, which was detected by CTPA or V/Q scan, and graded as disappearance, effective, no effect, or progressed to worse according to the following definitions:

Disappearance. $100 \%$ of the thrombus was resorbed.

Effective. $>25 \%$ of the thrombus resorbed.

No Effect. $<25 \%$ of the thrombus resorbed

Progressed to Worse. pulmonary embolism progressed to worse.

The principal safety outcome was bleeding, which included anticoagulant-induced bleeding, and liver or kidney dysfunction. Bleeding was defined and graded as (1) mild bleeding, which includes nasal bleeding, gum bleeding, skin bleeding or petechiae, blood-stained sputum, bleeding hemorrhoids, or hematuria under a microscope, and did not require a change in the therapeutic plan; (2) major bleeding, which included digestive system bleeding, gross hematuria for 2 continuous days, hemoptysis, and required to change the therapeutic plan; and (3) life-threatening or severe bleeding, which might cause cardiac or respiratory arrest, or require surgical intervention, including intracranial hemorrhage, anemia (hematocrit $<0.2$ ), and systolic blood pressure $<90 \mathrm{~mm} \mathrm{Hg}$.

2.4. Statistical Analysis. Data in this article were nonparametric except for the body weight and BMI. Statistical analysis was performed with SPSS (version 24). Measurement data were expressed as the mean \pm standard deviation; comparisons between groups were expressed by independent twosample $t$-test; categorical variables were expressed as a ratio and were assessed using the $\chi^{2}$ test; and the rank sum test was used to rank data. Comparisons of efficacy between two groups were analyzed by the Cox multivariate model. $P<0.05$ was considered statistically significant.

\section{Results}

3.1. Patients. From January 2015 through December 2016, 138 patients were enrolled to this study: 86 received rivaroxaban treatment, and 42 were given LMWH and warfarin (standard therapy) with 10 patients changing regimen between the first and fifth months. Table 1 summarizes the baseline demographics, treatment, and follow-up of patients in the current study. The mean age of patients in the rivaroxaban monotherapy group was higher than that in the standard therapy group. The ratio of intermediate-high-risk and PESI in the rivaroxaban monotherapy group was higher than that in the standard-therapy group. Other characteristics were not significantly different between the groups. 
TABLE 1: Demographic and clinical data of patients.

\begin{tabular}{|c|c|c|c|c|c|c|}
\hline Variable & Group & $N$ & Rivaroxaban & Warfarin & $\chi^{2} / T$ & $P$ value \\
\hline \multirow{2}{*}{ Sex } & $\mathrm{F}$ & 67 & $45(52.33 \%)$ & $22(52.38 \%)$ & \multirow{2}{*}{0.000} & \multirow{2}{*}{0.995} \\
\hline & M & 61 & $41(47.67 \%)$ & $20(47.62 \%)$ & & \\
\hline \multirow{2}{*}{ Combined with DVT } & Without & 69 & $48(55.81 \%)$ & $21(50 \%)$ & \multirow{2}{*}{0.384} & \multirow{2}{*}{0.536} \\
\hline & With & 59 & $38(44.19 \%)$ & $21(50 \%)$ & & \\
\hline \multirow{2}{*}{ HAS-BLED } & Lower danger & 122 & $85(98.84 \%)$ & $37(88.1 \%)$ & \multirow{2}{*}{7.288} & \multirow{2}{*}{$0.007 * *$} \\
\hline & Higher danger & 6 & $1(1.16 \%)$ & $5(11.9 \%)$ & & \\
\hline \multirow{4}{*}{ Risk stratification } & Lower danger & 27 & $22(25.58 \%)$ & $5(11.9 \%)$ & \multirow{4}{*}{0.384} & \multirow{4}{*}{0.536} \\
\hline & Intermediate-lower danger & 60 & $46(53.49 \%)$ & $14(33.33 \%)$ & & \\
\hline & Intermediate-high danger & 36 & $15(17.44 \%)$ & $21(50 \%)$ & & \\
\hline & Higher danger & 5 & $3(3.49 \%)$ & $2(4.76 \%)$ & & \\
\hline \multirow{2}{*}{ Expected course of treatment } & 3 months & 53 & $34(39.53 \%)$ & $19(45.24 \%)$ & \multirow{2}{*}{0.378} & \multirow{2}{*}{0.539} \\
\hline & 6 months & 75 & $52(60.47 \%)$ & $23(54.76 \%)$ & & \\
\hline \multirow{2}{*}{ Thrombolysis } & Without & 107 & $75(87.21 \%)$ & $32(76.19 \%)$ & \multirow{2}{*}{2.498} & \multirow{2}{*}{0.114} \\
\hline & With & 21 & $11(12.79 \%)$ & $10(23.81 \%)$ & & \\
\hline Age & - & 128 & $58.08 \pm 14.52$ & $52.55 \pm 14.80$ & 2.012 & $0.046 *$ \\
\hline Weight & - & $125^{\mathrm{a}}$ & $64.84 \pm 12.09$ & $61.76 \pm 12.06$ & 1.347 & 0.180 \\
\hline BMI & - & $125^{\mathrm{a}}$ & $24.28 \pm 3.61$ & $23.22 \pm 3.37$ & 1.583 & 0.116 \\
\hline PESI & - & 128 & $82.15 \pm 27.06$ & $71.83 \pm 24.34$ & 2.091 & $0.039 *$ \\
\hline sPESI & - & 128 & $1.02 \pm 1.10$ & $0.88 \pm 0.99$ & 0.711 & 0.478 \\
\hline
\end{tabular}

${ }^{a}$ Missing number is 3 .

TABLE 2: Efficacy outcomes.

\begin{tabular}{|c|c|c|c|c|c|c|}
\hline Variable & Group & $N$ & Rivaroxaban (86) & Warfarin (42) & $\chi^{2}$ & $P$ value \\
\hline \multirow{5}{*}{ Reexamination after 1 month } & Disappeared & 49 & $37(43.02 \%)$ & $12(28.57 \%)$ & 1.5396 & 0.2147 \\
\hline & Effective & 46 & $30(34.88 \%)$ & $16(38.1 \%)$ & 0.0810 & 0.7760 \\
\hline & No effect or progressed & 10 & $6(6.98 \%)$ & $4(9.52 \%)$ & 0.2343 & 0.6283 \\
\hline & Reappear & 0 & $0(0 \%)$ & $0(0 \%)$ & & \\
\hline & - & 23 & $13(15.12 \%)$ & $10(23.81 \%)$ & & \\
\hline \multirow{5}{*}{ Reexamination after 3 months } & Disappeared & 69 & $52(60.47 \%)$ & $17(40.48 \%)$ & 2.0916 & 0.1481 \\
\hline & Effective & 34 & $22(25.58 \%)$ & $12(28.57 \%)$ & 0.0950 & 0.7579 \\
\hline & No effect or progressed & 12 & $7(8.14 \%)$ & $5(11.9 \%)$ & 0.4267 & 0.5136 \\
\hline & Reappear & 0 & $0(0 \%)$ & $0(0 \%)$ & & \\
\hline & - & 7 & $5(5.81 \%)$ & $8(19.05 \%)$ & 0.0571 & 0.8111 \\
\hline
\end{tabular}

3.2. Clinical Outcome. As shown in Tables 2 and 3, the primary efficacy outcome was observed in 52 patients $(40.48 \%)$ in the rivaroxaban group compared with 17 patients $(60.47 \%)$ in the standard therapy group after 3 months $(P=0.033$, new data $P=0.148)$. According to the Cox multivariate model, there was no significant difference in efficacy outcomes between the two groups after 3-month treatment $(P=0.798,95 \%$ confidence interval $(\mathrm{CI}) 0.686$ to $1.336)$ or more than 6 -month treatment $(P=0.534,95 \% \mathrm{CI}$ 0.795 to 1.556$)$. Moreover, thrombus absorption was not significantly different within 1 month between the two groups
$(P=0.215)$. The rivaroxaban monotherapy group had a shorter hospitalization duration than that the standard therapy group, although this did not reach statistical significance. When the course of rivaroxaban monotherapy reached 6 months, we adjusted the dosage to $10 \mathrm{mg}$ once daily, and no decline in efficacy was found compared with standard therapy. Rather, their conditions improved after treatment for 6 months.

The principal safety outcome is shown in Table 4. Severe bleeding episodes were not observed in any patients in the rivaroxaban group compared with 3 patients 
TABLE 3: Efficacy in patients receiving the expected course of treatment.

\begin{tabular}{|c|c|c|c|c|c|c|}
\hline Duration & Efficacy & $N$ & Rivaroxaban (52) & Warfarin (23) & $\chi^{2}$ & $P$ value \\
\hline \multirow{4}{*}{1 month } & Disappeared & 27 & $22(42.31 \%)$ & $5(21.74 \%)$ & 1.8740 & 0.1710 \\
\hline & Effective & 30 & $20(38.46 \%)$ & $10(43.48 \%)$ & 0.1003 & 0.7514 \\
\hline & No effect or progressed & 6 & $4(7.69 \%)$ & $2(8.7 \%)$ & 0.0201 & 0.8874 \\
\hline & - & 12 & $6(11.54 \%)$ & $6(26.09 \%)$ & & \\
\hline \multirow{4}{*}{3 months } & Disappeared & 38 & $30(57.69 \%)$ & $8(34.78 \%)$ & 1.6519 & 0.1987 \\
\hline & Effective & 19 & $13(25 \%)$ & $6(26.09 \%)$ & 0.0074 & 0.9313 \\
\hline & No effect or progressed & 9 & $6(11.54 \%)$ & $3(13.04 \%)$ & 0.0301 & 0.8623 \\
\hline & - & 9 & $3(5.77 \%)$ & $6(26.09 \%)$ & & \\
\hline \multirow{4}{*}{6 months } & Disappeared & 39 & $31(59.62 \%)$ & $8(34.78 \%)$ & 1.8911 & 0.1691 \\
\hline & Effective & 19 & $11(21.15 \%)$ & $8(34.78 \%)$ & 1.1692 & 0.2796 \\
\hline & No effect or progressed & 7 & $5(9.62 \%)$ & $2(8.7 \%)$ & 0.0145 & 0.9043 \\
\hline & - & 10 & $5(9.62 \%)$ & $5(21.74 \%)$ & & \\
\hline
\end{tabular}

TABLE 4: Safety of patients with expected course of treatment.

\begin{tabular}{|c|c|c|c|c|c|c|}
\hline Variable & Group & $N$ & Rivaroxaban (86) & Warfarin (42) & $\chi^{2}$ & $P$ value \\
\hline \multirow{5}{*}{ Reexamination after 1 month } & No bleeding & 55 & $40(46.51 \%)$ & $15(35.71 \%)$ & 0.7656 & 0.3816 \\
\hline & Mild bleeding & 54 & $36(41.86 \%)$ & $18(42.86 \%)$ & 0.0066 & 0.9350 \\
\hline & Serve bleeding & 3 & $0(0 \%)$ & $3(7.14 \%)$ & 6.1429 & 0.0132 \\
\hline & Major bleeding & 2 & $0(0 \%)$ & $2(4.76 \%)$ & 4.0952 & 0.0430 \\
\hline & - & 14 & $10(11.63 \%)$ & $4(9.52 \%)$ & & \\
\hline \multirow{5}{*}{ Reexamination after 3 months } & No bleeding & 49 & $38(44.19 \%)$ & $11(26.19 \%)$ & 2.3872 & 0.1223 \\
\hline & Mild bleeding & 61 & $40(46.51 \%)$ & $21(50 \%)$ & 0.0721 & 0.7884 \\
\hline & Serve bleeding & 3 & $0(0 \%)$ & $3(7.14 \%)$ & 6.1429 & 0.0132 \\
\hline & Major bleeding & 2 & $0(0 \%)$ & $2(4.76 \%)$ & 4.0952 & 0.0430 \\
\hline & - & 13 & $8(9.3 \%)$ & $5(11.9 \%)$ & & \\
\hline \multirow{5}{*}{ Reexamination after 6 months } & No bleeding & 46 & $37(43.02 \%)$ & $9(21.43 \%)$ & 3.6617 & 0.0557 \\
\hline & Mild bleeding & 65 & $41(47.67 \%)$ & $24(57.14 \%)$ & 0.4982 & 0.4803 \\
\hline & Serve bleeding & 4 & $1(1.16 \%)$ & $3(7.14 \%)$ & 3.2292 & 0.0723 \\
\hline & Major bleeding & 3 & $1(1.16 \%)$ & $2(4.76 \%)$ & 1.5596 & 0.2117 \\
\hline & - & 10 & $6(6.98 \%)$ & $4(9.52 \%)$ & & \\
\hline
\end{tabular}

(7.14\%) in the standard therapy group $(P=0.0132)$. Major bleeding was observed in one patient $(1.16 \%)$ in the rivaroxaban group and two patients $(4.76 \%)$ in the standardtherapy group $(P=0.043)$. However, there were two cases of major hemorrhage (one case with abdominal hemorrhage and another with serve hemoptysis) leading to death in the standard-therapy group. The rate of all-cause mortality was $3.49 \%$ in the rivaroxaban group and $9.52 \%$ in the standard-therapy group.

Table 5 shows that the primary efficacy outcome and principal safety outcome were similar between groups except for the rate of all-cause mortality in PE patients with cancer, which was higher than that of the nontumor group (61.11\% vs $67.65 \%, P=0.762)$. Table 6 shows that efficacy outcome $(P=0.289)$ and safety outcome $(P=0.326)$ of rivaroxaban in patients with or without frailty were similar. For intermediate-high-risk patients, the efficacy of mono- therapy with rivaroxaban is similar to thrombolysis treatment (absorption of thrombosis rate was 63.64\% vs $46.15 \%, P=0.392$ ). And the incidence of bleeding was also similar in these 2 groups (minor bleeding $45.45 \%$ vs $76.92 \%, P=0.245$ ) (Table 7).

\section{Discussion}

Increasing numbers of phase III randomized clinical controlled trials have indicated that NOACs are not inferior to traditional standard therapy for the treatment of acute pulmonary embolism and have a better safety profile. Rivaroxaban was the first oral direct factor Xa inhibitor confirmed to be effective and safe in randomized controlled trials including EINSTEIN-DVT [9] and EINSTEIN-PE [10]. Westendrof et al. [11] evaluated the results of several real-world clinical studies including XALIA [12], XANTUS 
TABLE 5: Efficacy and safety of rivaroxaban in patients with pulmonary embolism combined with or without tumor.

\begin{tabular}{|c|c|c|c|c|c|c|}
\hline Duration & Efficacy & $N$ & PE with tumor (18) & PE without tumor (68) & $\chi^{2}$ & $P$ value \\
\hline \multirow{6}{*}{ Efficacy } & Disappeared & 57 & $11(61.11 \%)$ & $46(67.65 \%)$ & 0.0917 & 0.7620 \\
\hline & Effective & 16 & $2(11.11 \%)$ & $14(20.59 \%)$ & 0.6871 & 0.4072 \\
\hline & No effect & 5 & $1(5.56 \%)$ & $4(5.88 \%)$ & 0.0026 & 0.9592 \\
\hline & Progressed & 2 & $1(5.56 \%)$ & $1(1.47 \%)$ & 1.0212 & 0.3122 \\
\hline & Reappeared & 2 & $0(0 \%)$ & $2(2.94 \%)$ & 0.5294 & 0.4669 \\
\hline & - & 4 & $3(16.67 \%)$ & $1(1.47 \%)$ & & \\
\hline \multirow{6}{*}{ Bleeding } & No bleeding & 42 & $9(50 \%)$ & $33(48.53 \%)$ & 0.0063 & 0.9367 \\
\hline & Mild bleeding & 38 & 7 (38.89\%) & $31(45.59 \%)$ & 0.1446 & 0.7038 \\
\hline & Serve bleeding & 1 & $1(5.56 \%)$ & $0(0 \%)$ & 3.7778 & 0.0519 \\
\hline & Major bleeding & 1 & $0(0 \%)$ & $1(1.47 \%)$ & 0.2647 & 0.6069 \\
\hline & - & 4 & $1(5.56 \%)$ & $3(4.41 \%)$ & & \\
\hline & Death & 3 & $3(16.67 \%)$ & $0(0 \%)$ & 11.333 & $<0.001$ \\
\hline
\end{tabular}

TABLE 6: Efficacy and safety of rivaroxaban in patients with PE combined with or without frailty.

\begin{tabular}{|c|c|c|c|c|c|}
\hline & $N$ & PE with frailty (22) & PE without frailty $n=64$ & $\chi^{2}$ & $P$ value \\
\hline Efficacy & & & & -0.72 & 0.471 \\
\hline Disappeared & 55 & $12(54.55 \%)$ & $43(67.19 \%)$ & 1.35 & 0.289 \\
\hline Effective & 20 & $8(36.36 \%)$ & $12(18.75 \%)$ & 2.846 & 0.092 \\
\hline No effect & 5 & $1(4.55 \%)$ & $4(6.25 \%)$ & $<0.001$ & 1 \\
\hline Progressed & 2 & $0(0.00 \%)$ & $2(3.13 \%)$ & & 0.552 \\
\hline Bleeding & & & & -1.363 & 0.173 \\
\hline Bleeding & 39 & $8(36.36 \%)$ & $31(48.44 \%)$ & 0.963 & 0.326 \\
\hline Mild bleeding & 27 & $13(59.09 \%)$ & 27 (42.19\%) & 1.88 & 0.170 \\
\hline Serve bleeding & 1 & $0(0.00 \%)$ & $1(1.56 \%)$ & & 0.744 \\
\hline Major bleeding & 1 & $1(4.55 \%)$ & $0(0.00 \%)$ & & 0.256 \\
\hline
\end{tabular}

TABLE 7: Comparison of rivaroxaban and thrombolytic therapeutic in intermediate-high-risk patients.

\begin{tabular}{|c|c|c|c|c|c|}
\hline & & Thrombolysis (13) & Rivaroxaban (11) & $x^{2}$ & $P$ value \\
\hline \multirow[t]{6}{*}{ Efficacy } & & & & -1.479 & 0.139 \\
\hline & Disappeared & $6(46.15 \%)$ & $7(63.64)$ & 0.734 & 0.392 \\
\hline & Effective & $6(46.15 \%)$ & $2(18.18)$ & 1.028 & 0.311 \\
\hline & No effect & $0(0.00 \%)$ & $2(18.18)$ & & 0.199 \\
\hline & Progressed & $0(0.00 \%)$ & $0(0.00)$ & & \\
\hline & Reappeared & $1(7.69 \%)$ & $0(0.00)$ & & 0.542 \\
\hline \multirow[t]{6}{*}{ Bleeding } & & & & 2.517 & 0.113 \\
\hline & Bleeding & $3(23.08 \%)$ & $5(45.45 \%)$ & 0.524 & 0.496 \\
\hline & Mild bleeding & $10(76.92 \%)$ & $5(45.45 \%)$ & 1.354 & 0.245 \\
\hline & Serve bleeding & $0(0.00 \%)$ & $0(0.00 \%)$ & & \\
\hline & Major bleeding & $0(0.00 \%)$ & $0(0.00 \%)$ & & \\
\hline & Death & $0(0.00 \%)$ & $2(18.18 \%)$ & & 0.199 \\
\hline
\end{tabular}

[13], GARFIELD [13, 14], and ORBIT-AF [15] and reaffirmed the safety and efficacy of rivaroxaban previously observed in RCTs.

In China, a series of clinical trials of rivaroxaban for the treatment of pulmonary embolism were also reported [16-19] after it was approved for the treatment of acute pulmonary embolism in 2017. The results of these studies also indicated that rivaroxaban may be used as an alternative treatment to standard therapy for the treatment of acute pulmonary embolism, with indiscriminate efficacy and lower bleeding risk. Because these studies were all clinical studies with small sample sizes and therefore 
provided limited data, the current study has added to the domestic clinical evidence regarding rivaroxaban for the treatment of PE.

The main results of our study demonstrated that rivaroxaban monotherapy is not inferior to standard therapy for the treatment of pulmonary embolism, consistent with our previous findings and those of the EINSTEIN-PE study. The current study found that the efficacy of rivaroxaban increased with time, suggesting it might be more suitable for patients who need long-term anticoagulation treatment. Overall, 4832 patients were enrolled in the EINSTEIN study, where the main efficacy outcome was the occurrence of recurrent thrombotic events. Our study provides more specific information regarding efficacy, which can be divided into four levels: disappearance, effective, no effect, and progressed to worse according to imaging findings.

Apart from enhanced long-term efficacy for the treatment of acute pulmonary embolism, rivaroxaban had better safety than standard therapy. These results are consistent with previous large-scale RCT studies. Regarding recurrence rates, major bleeding rates, and mortality rates, this study, which revealed the true clinical level, was closer to the realworld XALIA study compared with other RCT studies. However, the XALIA study had no data for pulmonary embolism. Therefore, the data in the current study should provide support for clinical decision-making. The analysis of subgroups in acute pulmonary embolism was specific to this study and showed that rivaroxaban was comparable to standard treatment and LMWH for the treatment of frail patients, cancer patients, and high-risk patients, confirming evidence for its clinical application.

To ease the economic burden of patients, the dose of rivaroxaban was reduced to $10 \mathrm{mg}$ once daily for patients in the rivaroxaban monotherapy group who still need anticoagulation therapy for at least 3 months, and no decrease in efficacy was observed. There was a significant difference in efficacy between the two groups at 6 months, which suggested that for Chinese patients, after 3 months of treatment with $20 \mathrm{mg}$ once daily, a reduced dose of $10 \mathrm{mg}$ once daily can be effective.

This was a semiretrospective and semiprospective study and therefore had some limitations common to retrospective studies: (1) bias of patient memory, because the patients were diagnosed with $\mathrm{PE}$ during 3 years, many patients were already cured, and the degree of memory regarding bleeding events might be inaccurate; (2) bias of data integrity, most patients lacked records of CTPA or examination of urine at a specific timepoint; and (3) bias of loss of follow-up, the rate of lost to follow-up for the warfarin and rivaroxaban groups was $2.38 \%$ and $8.13 \%$, respectively. This study also included hospital admission bias, namely, patients hospitalized in the First Affiliated Hospital of Guangzhou Medical University do not fully represent the overall population of patients with acute pulmonary embolism treated in other hospitals.

In the subgroup analyses (frail patients, cancer patients, or intermediate-high risk patients) of this study, the number of cases was not large enough, and it will be necessary to conduct large-scale research to verify the results of this study.
Furthermore, because few patients with tumors were injected with LMWH subcutaneously in the clinic, we could not compare rivaroxaban monotherapy with LMWH therapy in tumor patients; therefore, it will be necessary to collect more LMWH therapy cases to conduct further research.

\section{Conclusions}

The efficacy of the rivaroxaban regimen was not inferior to the standard therapy. With an extension of treatment time, the rivaroxaban monotherapy group had a better efficacy and lower incidence of bleeding episodes compared with the standard-therapy group. The efficacy of rivaroxaban did not decrease when its dose was reduced to $10 \mathrm{mg}$ once daily.

\section{Abbreviations \\ APE: Acute pulmonary embolism \\ BMI: $\quad$ Body mass index \\ CTPA: Computed tomographic pulmonary angiography \\ INR: International normalized ratio \\ LMWH: Low-molecular-weight heparin \\ NOACs: Nonvitamin K-dependent new oral anticoagulants \\ NVAF: Nonvalvular atrial fibrillation \\ RCT: Randomized controlled trial.}

\section{Data Availability}

The data used to support the findings of this study are available from the corresponding author upon request.

\section{Ethical Approval}

This study was approved by the Ethics Committee of the First Affiliated Hospital of Guangzhou Medical University.

\section{Consent}

Prior to participation, all subjects must have provided written informed consent.

\section{Conflicts of Interest}

The authors declare that they have no competing interests. All other authors have reported that they have no relationships relevant to the contents of this paper to disclose.

\section{Authors' Contributions}

CL was the coordinator of the study, WH wrote the first draft of the manuscript. YH and LD were critically involved as coprincipal investigators in the planning and the conduct of the study (application for funding and trial design) and in finalizing the manuscript. TW and JW were involved in critically revising the manuscript. YG and YC helped to finalize the manuscript. XW and NZ were investigators at the clinical site. WH was responsible for planning all statistical analyses. All authors read and approved the final manuscript. 


\section{Acknowledgments}

This work was supported by grants from the Foundation of Guangzhou Medical University (B185004065), Department of Science and Technology of China grant 2016YFC1304102, and Science and Technology Program of Guangzhou, China 201604020185.

\section{References}

[1] M. D. Silverstein, J. A. Heit, D. N. Mohr, T. M. Petterson, W. M. O'Fallon, and L. J. Melton, "Trends in the incidence of deep vein thrombosis and pulmonary embolism: a 25 -year population-based study," Archives of Internal Medicine, vol. 158, no. 6, pp. 585-593, 1998.

[2] C. Kearon, E. A. Akl, J. Ornelas et al., "Antithrombotic therapy for VTE disease: CHEST guideline and expert panel report," Chest, vol. 149, no. 2, pp. 315-352, 2016.

[3] M. Mandalà and R. Labianca, "Venous thromboembolism (VTE) in cancer patients. ESMO Clinical Recommendations for prevention and management," Thrombosis Research, vol. 125, Suppl. 2, pp. S117-S119, 2010.

[4] R. D. Hull, G. F. Pineo, R. F. Brant et al., "Long-term lowmolecular-weight heparin versus usual care in proximal-vein thrombosis patients with cancer," The American Journal of Medicine, vol. 119, no. 12, pp. 1062-1072, 2006.

[5] J. A. Ross, M. M. Miller, and C. M. Rojas Hernandez, "Comparative effectiveness and safety of direct oral anticoagulants (DOACs) versus conventional anticoagulation for the treatment of cancer-related venous thromboembolism: a retrospective analysis," Thrombosis Research, vol. 150, pp. 86-89, 2017.

[6] M. N. Levine, C. Gu, H. A. Liebman et al., "A randomized phase II trial of apixaban for the prevention of thromboembolism in patients with metastatic cancer," Journal of Thrombosis and Haemostasis, vol. 10, no. 5, pp. 807-814, 2012.

[7] P. O. Hansson, J. Sorbo, and H. Eriksson, "Recurrent venous thromboembolism after deep vein Thrombosis," Archives of Internal Medicine, vol. 160, no. 6, pp. 769-774, 2000.

[8] J. A. Heit, D. N. Mohr, M. D. Silverstein, T. M. Petterson, W. M. O'Fallon, and L. J. Melton, "Predictors of recurrence after deep vein thrombosis and pulmonary embolism: a population-based cohort study," Archives of Internal Medicine, vol. 160, no. 6, pp. 761-768, 2000.

[9] R. Bauersachs, S. D. Berkowitz, B. Brenner et al., "Oral rivaroxaban for symptomatic venous thromboembolism," The New England Journal of Medicine, vol. 363, no. 26, pp. 2499-2510, 2010.

[10] H. R. Buller, M. H. Prins, A. W. Lensin et al., "Oral rivaroxaban for the treatment of symptomatic pulmonary embolism," The New England Journal of Medicine, vol. 366, no. 14, pp. 12871297, 2012.

[11] W. Ageno, L. G. Mantovani, S. Haas et al., "XALIA: rationale and design of a non-interventional study of rivaroxaban compared with standard therapy for initial and long-term anticoagulation in deep vein thrombosis," Thrombosis Journal, vol. 12, no. 1, p. 16, 2014.

[12] A. J. Camm, P. Amarenco, S. Haas et al., "XANTUS: a realworld, prospective, observational study of patients treated with rivaroxaban for stroke prevention in atrial fibrillation," European Heart Journal, vol. 37, no. 14, pp. 1145-1153, 2016.
[13] V. ten Cate, H. ten Cate, and F. W. A. Verheugt, "The Global Anticoagulant Registry in the FIELD-Atrial Fibrillation (GARFIELD-AF)," Netherlands Heart Journal, vol. 24, no. 10, pp. 574-580, 2016.

[14] J. P. Piccini, E. S. Fraulo, J. E. Ansell et al., "Outcomes registry for better informed treatment of atrial fibrillation: rationale and design of ORBIT-AF," American Heart Journal, vol. 162, no. 4, pp. 606-612.e1, 2011.

[15] J. Beyer-Westendorf, A. J. Camm, C. I. Coleman, and S. Tamayo, "Rivaroxaban real-world evidence: validating safety and effectiveness in clinical practice," Thrombosis and Haemostasis, vol. 116, Suppl. 2, pp. S13-s23, 2016.

[16] C. Zhang, Y. Huang, and L. Liang, "Observation of curative effect by rivaroxaban in the treatment of 20 pulmonary embolism cases," China Practical Medical, vol. 11, pp. 22-24, 2016.

[17] J. Bai, S. Qu, Y. Wang et al., "Observation of curative effect by rivaroxaban in the treatment of 20 pulmonary embolism cases," Xinjiang Medical Journal, vol. 43, no. 11, pp. 60-62, 2013.

[18] S. Liu, Y. Xu, J. Cai, and L. Guo, "Clinical observation and nursing intervention of rivaroxaban in the treatment of pulmonary embolism," China Pharmacy, vol. 27, no. 8, pp. 1115-1117, 2016.

[19] L. Tang and W. Zhao, "Curative effect of rivaroxaban in the treatment of acute pulmonary embolism," Journal of Clinical Pulmonary Medicine, vol. 19, no. 10, pp. 1780-1782, 2014. 